\title{
Cambios psicosociales, salud y calidad de vida de padres de niños con parálisis cerebral.
}

\author{
Psychosocial changes, health and quality of life of parents of children with cerebral palsy.
}

\author{
Edinson M. Perez ${ }^{1, a}$
}

\section{RESUMEN}

La parálisis cerebral es un transtorno de movimientos y posturas, además de otras disfunciones que limitan el desarrollo de actividades de la vida diaria; en niños, esta condición demanda cuidados y crianza especiales por parte de los padres, los cuales experimentan una serie de cambios y necesidades en sus ámbitos personal, familiar, laboral y social, desde el momento en que reciben el diagnóstico clínico y a lo largo del desarrollo del niño. En este proceso, los padres presentan frecuentemente síntomas de ansiedad, estrés, depresión, alteración del sueño y transtornos musculoesqueléticos, a todo lo cual se suma el bajo nivel de calidad de vida que experimentan. Varios estudios demuestran que los cambios psicosociales, de salud y calidad de vida de los padres son resultado tanto de las características del niño con parálisis cerebral, como de los rasgos personales de los padres y/o de factores ambientales y contextuales de la familia. Los sistemas de salud deben, por lo tanto asistir adecuadamente a los padres, en paralelo con el tratamiento de los niños afectados con parálisis cerebral y de acuerdo al contexto en el que se desenvuelven.

PALABRAS CLAVE: Parálisis cerebral, padres, cuidadores, calidad de vida, salud mental, dolor musculoesqueletico

\section{SUMMARY}

Cerebral palsy is a disorder of movements and postures, accompanied by other dysfunctions that limit the development of daily life activities; in children, this condition demands special care and upbringing to be provided by the parents. As a result, the latter experience a series of changes and needs in their personal, family, work and social spheres, from the moment they receive the clinical diagnosis and throughout the child's whole developmental process. As a result, parents frequently present symptoms of anxiety, stress, depression, sleep disturbances and musculoskeletal disorders, generating a low quality of life level. Several studies show that psychosocial changes, health and quality of life of parents are affected by the child's characteristics, the parents' personal traits and / or the family's environmental and contextual factors. It is suggested that the health systems must also assist the parents in parallel with the treatment of children with cerebral palsy, and according to the context in which they occur.

KEY WORDS: Cerebral palsy, parents, caregivers, quality of life, mental health, musculoskeletal pain.

\section{INTRODUCCIÓN}

La parálisis cerebral (PC) es un transtorno del movimiento y postura, con frecuencia se acompaña con otras disfunciones de tipo sensoriales, perceptuales, cognitivas, conductuales y en algunos casos con transtornos convulsivos (1-3); este conjunto de disfunciones impide al niño a participar en actividades esenciales para su vida como el autocuidado, juego, educación, comunicación y otras de carácter social, las

1 Hospital II Lima Norte Callao "Luis Negreiros Vega". Lima, Perú.

a Tecnólogo Médico Terapeuta Ocupacional 
limitaciones se acentúan con la presencia de dolor y severidad motora $(2,4)$.

De otra parte, las alteraciones que caracterizan al niño con PC demandan una crianza y cuidados especiales por parte de los padres (papá y mamá) y de otros integrantes del entorno familiar (3), quienes al mismo tiempo de experimentar cambios en sus vidas, tienen un impacto en su salud y calidad de vida (CV) a lo largo del desarrollo del niño (5). Estos aspectos de los padres/cuidadores no son atendidos en forma paralela al tratamiento de los niños con PC en los sistemas de salud del Perú, a esto se suma una escases de investigaciones nacionales referentes a las necesidades de los padres de niños con PC.

El objetivo de este trabajo es revisar literatura sobre los cambios psicosociales de mayor énfasis, las afecciones de salud de mayor frecuencia y la variación de la CV de los padres de niños con PC a nivel mundial. Para esto se hizo una búsqueda bibliográfica no sistemática en las siguientes bases de datos: ProQuest, Scielo y PubMed. Luego, se seleccionó artículos originales y de revisión de acceso libre en idioma español, portugués e inglés relevantes a los objetivos de este trabajo.

\section{Cambios psicosociales en los padres de niños con parálisis cerebral.}

El nacimiento de un hijo en condiciones saludables genera experiencias diferentes en hombres y mujeres, donde las expectativas y simbolismos de ser papá o mamá están influenciadas por la representación social que adquieren en el contexto social en el cual se desarrollan (6). Con este evento la familia vivencia cambios en su funcionamiento que implica, aprendizaje de los padres en el cuidado del hijo, en compartir tareas domésticas con el conyugue y en asumir otras responsabilidades (7).

Pero cuando los padres tienen un niño con PC, la vida familiar se perturba, reflejándose desde la confirmación del diagnóstico clínico del niño, situación que los envuelve en un proceso de duelo, que implica un estado de confusión, de sensación de pérdida del hijo esperado, de negación de la condición de su hijo, de rabia y sentimientos de culpa hasta que finalmente logran afrontar la realidad que les depara la vida (8).

Estudios cualitativos han revelado que los padres después del diagnóstico de PC de sus hijos afrontaron dificultad para asimilar la noticia en el corto plazo y percibieron que la orientación sobre la afectación del niño en la mayoría de veces no fue suficiente por parte del personal de salud $(9,10)$.

En otros contextos, la percepción de información recibida sobre el problema que afecta al niño resultó positiva, tal como lo demostró un estudio cuantitativo transversal en 30 madres de niños con PC, en el cual la mayoría reportó haber recibido del personal de salud información sobre la afectación del niño y lo consideraron de nivel suficiente o regular, mientras que el $6,66 \%$ consideraron de nivel insuficiente, además en este mismo estudio el $86,66 \%$ de las madres reportaron estar bien informadas sobre la condición del niño (11).

Por otra parte, con el desarrollo del niño los padres dedican tiempo y esfuerzo, experimentando nerviosismo y miedo de no hacer todo lo necesario para el bienestar y futuro de su hijo, aumentando de este modo sus preocupaciones parentales $(10,12)$, pero con el transcurrir del tiempo, algunos llegan a adaptarse a diferentes demandas de cuidado (9), incluso sin medir la afectación de su propio estado de salud (10) y desatendiendo asuntos importantes para el funcionamiento familiar, como la actividad laboral, que es abandonada por uno de los padres (con frecuencia la mamá) para dedicarse al cuidado del hijo y dejando de priorizar algunos requerimientos de otros integrantes de la familia (9-11).

En un estudio transversal en 52 padres de niños con PC, los investigadores reportaron que los problemas que experimentaron los padres con la crianza del niño fueron: perdida de trabajo, perdida de disfrute familiar, problemas de concentración y problemas financieros, estos últimos fueron los de mayor frecuencia (13).

En un estudio cualitativo en 17 padres de niños con PC, Alaee et al., mostraron aspectos psicoemocionales de los padres durante el cuidado del niño con PC, estos fueron agrupados en tres subcategorías: estados de preocupación sobre el niño, conflictos intrapersonales con respecto al niño y a ellos mismos, y sensaciones de soledad frente al cuidado del niño, este último se enfatizó cuando la condición del niño era de tipo severo (12).

Otra dificultad que suelen enfrentar los padres, es la afectación de las relaciones de pareja, manifestándose en conflictos frecuentes que pueden llegar incluso a separación de los padres, mucho más si estos ya 
tenían problemas antes del diagnóstico de $\mathrm{PC}$ en el niño $(8,10,11)$. Además, el funcionamiento familiar y satisfacción marital en los padres evidencia afectación mucho más cuando la condición neurológica del niño cursa con gravedad moderada y severa en comparación con los de gravedad leve (14), esto no debe descartar la situación contraria de algunos padres que viven la afectación del niño como la oportunidad de fortalecer las relaciones de pareja y familiares.

A nivel social los padres con frecuencia están expuestos a cuestionamientos por la ignorancia de las personas acerca del problema del niño con PC, tienen limitación para participar en actividades sociales por la demanda de cuidado hacia el niño o por que la sociedad no siempre los considera en eventos sociales, esta situación hace que algunos padres opten por el confinamiento en casa junto a su hijo (12).Un estudio mixto (cuantitativo transversal y cualitativo con enfoque fenomenológico) realizado en padres/ cuidadores de niños con PC, encontró en la parte cualitativa que la mayoría de los padres necesitan ayuda para el cuidado en casa y atención de salud domiciliaria, de tal manera que puedan realizar otras actividades personales o participar de actividades sociales (15).

En efecto, un estudio cualitativo con enfoque fenomenológico en 15 mamás de niños con PC encontró que el apoyo familiar y social para el cuidado del niño resultó en una percepción positiva y de disfrute en la mayoría de las mamás, a pesar que el desarrollo de sus actividades cotidianas se vieron afectadas (16). La asistencia a los padres frente a la discapacidad del niño tiene incluso beneficios para contrarrestar los embates del estrés a los que se ven sometidos en el día a día (17).

Por otra parte, un estudio cualitativo realizado en 6 familias de niños con PC que vivieron en un contexto de vulnerabilidad social experimentaron carencia económica y dificultades para acceder a bienes y servicios tanto públicos como privados, esta condición les exigió reorientar las prioridades de las necesidades y en la mayoría de los casos les generó angustia por no conseguir todos los requerimientos básicos para atender al niño afectado y a los demás integrantes de la familia (9).

Frente al contexto de vulnerabilidad social, el estudio anterior también mostró que las familias de niños con PC argumentaron la necesidad de reorganizar las relaciones y rutinas, de motivarse a buscar información sobre las características y manejo del niño para crear sus propias estrategias de cuidado y no depender totalmente de los profesionales de salud, pero a pesar de esto las familias encontraron otras dificultades como la de accesibilidad a entornos físicos tanto para la movilización del niño dentro del hogar como en los entornos públicos (9). Con respecto a la accesibilidad a entornos fiscos, el estudio invita a suponer que en los contextos de vulnerabilidad social, los padres necesitan adaptaciones en los ambientes del hogar que faciliten la ejecución de las actividades de la vida diaria del niño de forma práctica y segura; mientras que en los entornos públicos, necesitan gestión de adecuación urbanística para el uso de silla de ruedas $u$ otras ayudas para la marcha.

Deotraparte, cuando el familiar(que frecuentemente es la mamá) del niño con PC no encuentra las condiciones de un entorno físico facilitador, se ve en la necesidad de cargar al niño o someterse a un confinamiento en casa para evitar mayor fatiga de la que va teniendo por la atención diaria al hijo y por las tareas del hogar que adicionalmente realiza $(9,18)$.

\section{Salud de los padres de niños con parálisis cerebral}

Las características disfuncionales del niño con PC es una condición crónica que exige un cuidado a largo plazo, dicha condición del niño genera un desgaste físico y mental en el cuidador primario, cuyos síntomas frecuentes a nivel mental son ansiedad y depresión $(5,19,20)$.

Los síntomas de ansiedad y depresión además de ser frecuente en los padres/cuidadores de niños con PC, impactan negativamente en el bienestar de los niños, tal como lo demostró un estudio cuantitativo transversal realizado en 97 niños con PC y su mamás, en dicho estudio los investigadores encontraron que las puntuaciones altas (mayor afectación) del inventario de ansiedad de Beck en las mamás tuvieron correlación negativa con el dominio físico de calidad de vida de los niños, mientras que las puntuaciones altas (mayor afectación) del inventario de depresión de Beck en las mamás tuvieron correlación negativa con el dominio psicosocial de calidad de vida de los niños (21).

Además de los síntomas de ansiedad y depresión, hay factores psicosociales que afectan la salud mental de los padres de niños con PC. Al respecto, los resultados de un estudio transversal en 91 padres de niños con discapacidad física, donde el $82 \%$ de estos 
Cambios psicosociales, salud y calidad de vida de padres de niños con parálisis cerebral.

padres tuvieron niños con PC, encontró que el $44 \%$ de los padres tuvieron síntomas psiquiátricos, en este grupo de padres los factores que se relacionaron a pobre salud mental fueron por una parte la dificultad de los niños para caminar y realizar las actividades de vida diaria, mientras que de otra parte estuvieron los bajos ingresos familiares, falta de creencia religiosa, síntomas de distres parental y pobre interacción padreniño (22).

De manera particular, un estudio transversal en 45 mamás de niños con PC, analizó los problemas psicosociales y depresión, los resultados mostraron que el $89 \%$ de las mamás tuvieron síntomas de depresión desde un grado mínimo hasta moderado; en cuanto a los problemas psicosociales, el $76 \%$ presentaron desde leve a severa adversidad psicosocial; además encontraron que las puntuaciones de adversidad psicosocial se correlacionaron positivamente con las puntuaciones de depresión y las variables que tuvieron una fuerte asociación con la depresión de las mamás fueron: pobre relación de pareja, pobre interacción social y condiciones económicas adversas (23).

Otro de los síntomas que afecta la salud de los padres de niños con PC es el estrés, tal como lo evidenció un estudio transversal en 276 padres de niños con PC, en cuyos resultados los niveles de estrés en las mamás fue mayor en comparación al de los papás (24). También se puede apreciar en otro estudio transversal realizado en 285 mamás de niños con problemas físicos crónicos, sensoriomotores-mentales y psicológicos, que los niveles de estrés en las mamás de niños con problemas sensoriomotor-mentales que incluye $\mathrm{PC}$ fue mayor que en las mamás de niños con afectación psicológica y de aquellas que tienen enfermedades físicas crónicas como leucemia, diabetes o problemas renales (25).

En un estudio transversal realizado en una muestra de 223 mamás de niños y adolescentes con PC, los investigadores encontraron que el $45.3 \%$ de las mamás tuvieron altos niveles de estrés y proporcionalmente las mamás de niños grandes con PC (entre 9 y 11 años) tuvieron mayores niveles de estrés que las mamás de otros grupos de edades; en este estudio también mostraron que las mamás de niños con PC severa en el área motora tuvieron mayores niveles de estrés que las mamás de niños con afectación leve (26). Pero eso no significa que la edad mayor y gravedad motora del niño con PC implique necesariamente una asociación con altos niveles estrés en las mamás.
Además, un estudio de revisión mostró que las alteraciones psíquicas y problemas conductuales del niño con PC frecuentemente están relacionados a altos niveles de estrés en los padres, mientras que el buen funcionamiento familiar, el apoyo social, estrategia de afronte al cuidado y participación en actividades sociales están relacionados con la reducción de los niveles de estrés (27).

En relación a la salud física, un estudio transversal en 100 mamás y sus hijos con PC, encontró que las mamás de niños con PC proporcionalmente presentaron mayores síntomas de dolor y disfunción a nivel de cuello, espalda baja y miembro superior que limitaron el desarrollo de sus actividades cotidianas (28). También un estudio transversal en 180 mamás de niños con PC reportó que el $80.6 \%$ tiene más de una condiciones crónica y las más frecuentes son dolor a nivel de espalda, migrañas y alteraciones de sueño (29). Algo similar encontró un estudio comparativo en 85 mamás de niños con PC y 42 mamás de niños saludables, en el cual la presencia de dolor a nivel de cuello, espalda baja o en alguna región corporal fue mayor en mamás de niños con PC, este mismo estudio reportó que las mamás de niños con PC que experimentaron dolor tuvieron significativamente mayor edad que las mamás sin dolor, además el estado funcional del niño con PC en las mamás que experimentaron dolor fue significativamente peor que los niños de mamás que no experimentaron dolor (30). Esto no necesariamente significa que la edad de la mamá del niño con PC o el estado funcional del niño con $\mathrm{PC}$ sean factores que condicionen a la experiencia de dolor en las mamás, pues hay otros factores a nivel del niño con PC, ambientales y ocupacionales de las mamás que pueden influenciar en la experiencia de dolor físico.

Los familiares cuidadores de personas con PC en un estudio cualitativo reportaron alteración de la salud física general y sensaciones de dolor físico (31), estas alteraciones de salud se expresan a menudo con afectación a nivel de columna, disfunciones estomacales y condiciones físicas crónicas que contribuyen en la afectación de la salud mental (27).

Por tanto la salud física y mental suele afectarse en la mayoría de padres y/o cuidadores de niños con PC y algunas veces podrían estar asociados a algunas características del niño o de los padres, sin descartar factores contextuales en el cual se desarrolla la familia. 


\section{Calidad de Vida de los padres de niños con parálisis cerebral}

La calidad de vida (CV) es un concepto amplio sobre la evaluación de la satisfacción que tiene la persona en lo que va consiguiendo materialmente y de lo que va experimentando en cada momento de su vida $(32,33)$. Además, para la Organización Mundial de la Salud las evaluaciones de satisfacción están mediadas por los valores y contextos culturales en el que vive la persona, resultando la $\mathrm{CV}$ en la percepción de bienestar a nivel de cuatro principales dimensiones: físico, psicológico, social y ambiental, aunque bajo la perspectiva de otros modelos de $\mathrm{CV}$ las dimensiones varían, sin dejar su esencia subjetiva $(34,35)$.

Y en relación a los padres, se puede observar que tienen una serie de experiencias en cada momento del desarrollo del niño con PC, incluso tienen que lidiar con un entorno social que suele desconocer la situación del niño (9). No obstante, los padres ven afectados aspectos laborales, económicos, sociales, salud física y mental, al mismo tiempo que demandan acceso a entornos y servicios públicos para el niño y la familia (10).

Esta situación de los padres de los niños con PC supone una afectación de la CV (13) que se evidencia en una serie de estudios, tal como lo demostró Pousada et al., en una revisión sistemática y en el que encontró tres grupos de factores relacionados con el impacto de cuidado del niño con PC que afectan la CV de los padres (36), estos son:

- El primer grupo de factores corresponde a las características del niño en el cual incluyen la afectación motora (aunque en los estudios hay resultados contrarios en este aspecto), problemas conductuales, cognitivos y de comunicación.

- El segundo grupo de factores corresponde a las características de la familia y aquí se mencionan los bajos niveles de autoeficacia y autopercepción de los padres, pobre administración del tiempo, deficiente control del estrés, sobreprotección al niño, débil adaptación marital, poco apoyo familiar, alteraciones en el funcionamiento familiar y diferencia de género en asumir roles de cuidado.

- El tercer grupo hace referencia a los factores contextuales señalando el apoyo social como un moderador entre la discapacidad del niño y el bienestar de los padres.
El cuidado de un niño con $\mathrm{PC}$ puede afectar la $\mathrm{CV}$ de los padres en uno o más dominios. Al respecto, Zanon y Batista en un estudio transversal encontraron que la $\mathrm{CV}$ en 82 cuidadores de niños con PC se afectó más en los dominios de vitalidad y rol emocional según el cuestionario de salud 36 en su versión corta de la Medical Outcomes Study (SF- 36) aunque otros dominios también estuvieron bajos como el de salud general y percepción del dolor, mientras que el menos afectado fue la función física (20).

Algo contrario se observa en un estudio de caso control realizado en 42 mamás tanto de niños con PC como de niños con desarrollo normal, en el que también utilizaron el SF- 36 para medir la CV, este estudio encontró que la $\mathrm{CV}$ de las mamás de niños con PC tuvieron puntajes bajos en todos los dominios, afectándose en mayor medida el rol físico y función física, mientras que el menos afectado fue el rol emocional (37).

Utilizando un instrumento diseñado para tal investigación Mohammed et al (38) encontraron que la $\mathrm{CV}$ de 65 cuidadores de niños con PC fue baja en el $63,1 \%$ de ellos, en este estudio el $92,3 \%$ de los cuidadores fueron mamás de los niños con PC y el $89,2 \%$ del total de cuidadores se dedicó a tareas del hogar. Además, los dominios de CV estudiados fueron físico, apoyo para el cuidado, bienestar social y carga financiera, estos dos últimos fueron los más afectados en los cuidadores en el $75,4 \%$ y $81,5 \%$ respectivamente; también encontraron que $53,8 \%$ de los cuidadores obtuvo un alto nivel en el dominio de apoyo para el cuidado, mientras que el tipo de ocupación del cuidador fue el único factor que afectó significativamente su CV general. Este estudio, además de mostrar la afectación de $\mathrm{CV}$, muestra que el cuidado del niño con PC y el tipo de ocupación del cuidador son factores que influyen de forma contraria en la percepción de CV.

Por otra parte, la CV de los padres del niño con PC podría verse afectada por la gravedad de la función motora del niño, en este sentido los estudios han mostrado resultados contradictorios.

Surender et al (39) en un estudio transversal encontraron que el $67 \%$ de los niños con PC y sus familiares/cuidadores tuvieron afectado su $\mathrm{CV}$ desde un nivel leve a severa, la afectación estuvo relacionada con la gravedad de la función motora del niño, y los dominios más afectados fueron independencia física, movilidad, carga clínica e integración social. 
Cambios psicosociales, salud y calidad de vida de padres de niños con parálisis cerebral.

Glinac et al., en un estudio comparativo de 71 mamás de niños con PC y 70 mamás de niños saludables encontraron que a mayor afectación del funcionamiento motor del niño con PC hubo peor CV en sus mamás, siendo los dominios de funcionamiento social, actividades diarias, funcionamiento parental y funcionamiento familiar los más afectados (40).

De modo similar, Basaran et al., en un estudio comparativo de 143 cuidadores de niños con PC $(96,5 \%$ padres) y 60 cuidadores de niños sin discapacidad, encontraron que la $\mathrm{CV}$ de los cuidadores de niños con PC fue baja en comparación con el grupo control, y se afectó en mayor medida los dominios físico y psicológico según el instrumento WHOQOLBREF de la OMS (41). Además, demostraron que la afectación de la CV de los cuidadores de niños con PC tiene una relación significativa con la limitación de la función motora gruesa del niño.

En esta línea de investigación, Carvalho et al., en un estudio transversal en 31 mamás de niños con PC utilizaron el WHOQOL-BREF como instrumento de medición de $\mathrm{CV}$ y en sus resultados mostraron que la mayoría de mamás de niños con afectación motora grave percibieron negativamente los dominios de relaciones sociales y medio ambiente, mientras que las mamás de niños con afectación motora moderada en su mayoría percibieron negativamente el dominio medio ambiente, y finalmente las mamás de niños con afectación motora leve en su mayoría percibieron negativamente la dimensión física (42). Todas las otras dimensiones fueron percibidas positivamente por la mayoría de mamás en los tres grupos de afectación motora (Leve, moderada y severa) del niño.

Con respecto a la gravedad de la función motora del niño algunos estudios han encontrado que no se relaciona con la afectación de la $\mathrm{CV}$ en los padres $(28,38)$.

La edad es otra característica del niño con PC que tiene evidencia controversial en la afectación de la CV de sus padres, unos estudios encontraron que a mayor edad del niño con PC hay peor $\mathrm{CV}$ en sus padres $(42,43)$, mientras que otros estudios demostraron que la edad del niño con PC no tuvo relación con la afectación de la CV de sus padres $(38,39)$.

La literatura también muestra que las alteraciones de salud afectan la CV de los padres de niños con PC. A nivel físico, los transtornos musculoesqueléticos en espalda baja, cuello y hombro-brazo mostraron afectación de la mayoría de dominios de CV de los padres (28), a esto se suma condiciones crónicas como migraña y transtornos de sueño que se comportaron como determinantes significantes de afectación de la CV relacionada a la salud en el dominio físico (29). Mientras que a nivel mental se encontró evidencia de síntomas de ansiedad y depresión al mismo tiempo que baja CV en los padres de niños con PC (20). Esto sugiere que podría haber una relación entre estas variables.

Adicionalmente algunos estudios mostraron que elevados niveles de estrés se relacionaron con una alteración en la $\mathrm{CV}$ de los padres de niños con PC $(29,43)$.

Por otra parte, las investigaciones también han reportado aunque escasamente que la mejoría de los niños con PC después de un proceso de tratamiento rehabilitador no impactaron positivamente en la mejora de la CV de sus padres $(44,45)$.

Pero lo que evidenció ser un contribuyente en la mejora de la $\mathrm{CV}$ de los padres de niños con PC, fueron por una parte el apoyo para el cuidado del niño (38) y de otra parte el apoyo social a los padres, este último mostró un impacto positivo en el dominio mental de la $\mathrm{CV}$ y a su vez se relacionó con una disminución en el estrés de los padres (43). En tal sentido el apoyo social se comportó como un factor protector en los cuidadores de niños con PC frente al riesgo de estrés (46), en consecuencia se puede suponer que los padres que experimentan apoyo social tienen menos síntomas de estrés y mejor percepción de su CV.

\section{CONCLUSIONES}

Finalmente, se puede sostener que el niño con PC condiciona a los padres no solo a una serie de cambios en sus vidas, sino también a necesidades especiales para cumplir con el cuidado y crianza del hijo. En este contexto se aprecia alteración de la salud de los padres manifestándose con síntomas de ansiedad, estrés, depresión, alteraciones de sueño y transtornos musculoesqueléticos.

Por otro lado, los estudios muestran que los padres de niños con PC tienen baja CV y que la afectación de sus dominios son influenciados por las características del niño, de los padres y/o factores ambientales/ contextuales en el cual se desarrolla la familia, esto sugiere a los sistemas de salud de nuestro país, no solo a proponer estrategias de intervención para el niño sino también para los padres, de tal forma que estos 
mejoren su salud y CV, en consecuencia puedan darle mejor cuidado y $\mathrm{CV}$ al niño que día a día sufre los embates de la PC.

\section{Correspondencia:}

Edinson M. Perez

Jr. San Gabriel No 249, Urb. San Carlos, Comas, Lima, Perú

Correo electrónico: edinson.mpa@gmail.com

\section{REFERENCIAS BIBLIOGRÁFICAS}

1. Colver A, Fairhurst C, Pharoah POD. Cerebral palsy. The Lancet. 2014; 383(9924):1240-9.

2. Sanger TD. Movement Disorders in Cerebral Palsy. Journal of Pediatric Neurology. 2015;13(4):198207.

3. Muriel V, Ensenyat A, García-Molina A, AparicioLópez C, Roig-Rovira T. Déficits cognitivos y abordajes terapéuticos en parálisis cerebral infantil. Acción Psicológica. 2014;11(1):107-17.

4. Fauconnier J, Dickinson HO, Beckung E, Marcelli M, McManus V, Michelsen SI, et al. Participation in life situations of 8-12 year old children with cerebral palsy: cross sectional European study. BMJ. 2009;338:1458.

5. Barrera L, Sánchez B, Carrillo GM. La carga del cuidado en cuidadores de niños con enfermedad crónica. Revista Cubana de Enfermería. 2013;29(1):39-47.

6. Puyana Y, Mosquera C. Traer "hijos o hijas al mundo": significados culturales de la paternidad y la maternidad. Revista Latinoamericana de Ciencias Sociales, Niñez y Juventud. 2005; 3(2):111-40.

7. Jager ME, Bottoli C. Paternidade: vivência do primeiro filho e mudanças familiares. Psicologia: teoria e prática. 2011;13(1):141-53.

8. Madrigal A. Familias ante la parálisis cerebral. Psychosocial Intervention. 2007;16(1):55-68.

9. Baltor MRR, Dupas G. Experiences from families of children with cerebral paralysis in context of social vulnerability. Revista Latino-Americana de Enfermagem. 2013; 21(4):956-63.

10. Fernández M, García M, Berrocal M, Benítez A, Robles C, Laynez C. Experiences and changes in parents of children with infant cerebral palsy: a qualitative study. An Sist Sanit Navar. 2013;36(1):9-20.

11. Pérez L, Mendiondo P. La Parálisis cerebral infantil: $\mathrm{Su}$ impacto sobre el ajuste emocional y desempeño social de la madre. Revista Archivo
Médico de Camagüey. 2007;11(5):0-0.

12. Alaee N, Shahboulaghi FM, Khankeh H, Mohammad Khan Kermanshahi S. Psychosocial Challenges for Parents of Children with Cerebral Palsy: A Qualitative Study. Journal of Child and Family Studies. 2015;24(7):2147-54.

13. Olawale O, Deih A, Yaadar R. Psychological impact of cerebral palsy on families: The African perspective. Journal of Neurosciences in Rural Practice. 2013;4(2):159-63.

14. Bemister TB, Brooks BL, Dyck RH, Kirton A. Parent and family impact of raising a child with perinatal stroke. BMC Pediatrics. 2014;14:182.

15. Chiluba BC, Moyo G. Caring for a cerebral palsy child: a caregivers perspective at the University Teaching Hospital, Zambia. BMC Res Notes. 2017;10(1):724.

16. Glasscock R. A phenomenological study of the experience of being a mother of a child with cerebral palsy. Pediatric Nursing; Pitman. 2000; 26(4):407-10.

17. Hallberg U. Differences in health and well-being of parents of children with disabilities. Int J Qual Stud Health Well-being. 2014;9:0-0. doi: 10.3402/ qhw.v9.24343

18. Dambi JM, Jelsma J, Mlambo T. Caring for a child with Cerebral Palsy: The experience of Zimbabwean mothers. African Journal of Disability. 2015;4(1):1-10.

19. Marx C, Rodrigues EM, Rodrigues MM, Vilanova LCP. Depression, anxiety and daytime sleepiness of primary caregivers of children with cerebral palsy. Revista Paulista de Pediatria. 2011;29(4):483-8.

20.Zanon MA, Batista NA. Quality of life and level of anxiety and depression in caregivers of children with cerebral palsy. Revista Paulista de Pediatria. 2012;30(3):392-6.

21. Türkoglu S, Bilgiç A, Türkoglu G, Yilmaz S. Impact of symptoms of maternal anxiety and depression on quality of life of children with cerebral palsy. Noro-Psikyatri Arsivi. 2016;53(1):49-54.

22. Hung J-W, Wu Y-H, Chiang Y-C, Wu W-C, Yeh C-H. Mental health of parents having children with physical disabilities. Chang Gung Med J. 2010;33(1):82-91.

23. Badaru UM, Ogwumike OO, Adeniyi AF, Kaka B. Psychosocial adversities and depression in mothers of children with cerebral palsy in Nigeria. Journal of Pediatric Neurology. 2013;11(1):1-7.

24. Dahlbeck DT. Life satisfaction and stress among mothers and fathers of children with cerebral palsy: The impact of social support, financial 
Cambios psicosociales, salud y calidad de vida de padres de niños con parálisis cerebral.

stress, positive affect, relationship satisfaction, and religious community support. Tesis de Ph.D. United States,Tennessee: The University of Memphis; 2009.

25. Feizi A, Najmi B, Salesi A, Chorami M, Hoveidafar R. Parenting stress among mothers of children with different physical, mental, and psychological problems. J Res Med Sci. 2014;19(2):145-52.

26. Ribeiro MFM, Sousa ALL, Vandenberghe L, Porto CC. Parental stress in mothers of children and adolescents with cerebral palsy. Revista Latino-Americana de Enfermagem. 2014;22 (3):440-7.

27. Ribeiro MFM, Porto CC, Vandenberghe L. Parental stress in families of children with cerebral palsy: An Integrative Review. Ciência \& Saúde Coletiva. 2013;18(6):1705-15.

28. Kavlak E, Altuğ F, Büker N, Şenol H. Musculoskeletal system problems and quality of life of mothers of children with cerebral palsy with different levels of disability. J Back Musculoskelet Rehabil. 2015;28(4):803-10. doi: 10.3233/BMR150588

29. Lee MH, Matthews AK, Park C. Determinants of Health-related Quality of Life Among Mothers of Children With Cerebral Palsy. J Pediatr Nurs. 2019;44:1-8. doi: 10.1016/j.pedn.2018.10.001

30. Terzi R, Tan G. Musculoskeletal system pain and related factors in mothers of children with cerebral palsy. Agri. 2016;28(1):18-24.

31. Wittenberg E, Saada A, Prosser LA. How illness affects family members: A qualitative interview survey. The Patient. 2013;6(4):257-68.

32. Fernández-Mayoralas G, Rojo F. Calidad de vida y salud: planteamientos conceptuales y métodos de investigación. Territoris. 2005; 5:117-36.

33. Nava G. La calidad de vida: Análisis multidimensional. Enf Neurol (Mex). 2012;11(3):129-37.

34. Salas Z. C, Garzón D. MO. La noción de calidad de vida y su medición. Revista CES Salud Pública. 2013;4(1):36-46.

35. Urzúa M A, Caqueo-Urízar A. Calidad de vida: Una revisión teórica del concepto. Terapia psicológica. 2012;30(1):61-71.

36. Pousada M, Guillamón N, Hernández-Encuentra E, Muñoz E, Redolar D, Boixadós M, et al. Impact of Caring for a Child with Cerebral Palsy on the Quality of Life of Parents: A Systematic Review of the Literature. J Dev Phys Disabil. 2013;25(5):54577.

37. Borzoo S, Nickbakht M, Jalalian M. Effect of child's cerebral palsy on the mother: a case control study in Ahvaz, Iran. Acta Facultatis Medicae Naissensis. 2014;31(1):75-9.

38. Mohammed F, Ali S, Mustafa M. Quality of life of cerebral palsy patients and their caregivers: A cross sectional study in a rehabilitation center Khartoum-Sudan (2014 - 2015). J Neurosci Rural Pract. 2016. 7(3): 355-361. doi: 10.4103/09763147.182778

39. Surender S, Gowda V, Sanjay K, Basavaraja G, Benakappa N, Benakappa A. Caregiver-reported health-related quality of life of children with cerebral palsy and their families and its association with gross motor function: A South Indian study. J Neurosci Rural Pract. 2016; 7(2): 223-227. doi: 10.4103/0976-3147.178657

40. Glinac A, Matović L, Delalić A, Mešalić L. Quality of Life in Mothers of Children with Cerebral Palsy. Acta clinica Croatica. 2017;56(2.):299-307.

41. Basaran A, Karadavut KI, Uneri SO, Balbaloglu $\mathrm{O}$, Atasoy N. The effect of having a children with cerebral palsy on quality of life, burn-out, depression and anxiety scores: a comparative study. Eur J Phys Rehabil Med. 2013;49(6):81522.

42. Carvalho JT de M, Rodrigues NM, Silva LVC da, Oliveira DA de. Life quality of mothers with teenagers and children victims of cerebral palsy. Fisioterapia em Movimento. 2010; 23(3):389-97.

43. Mouilly M, Faiz N, Ahami AOT. The quality of life of parents with children and adolescents suffering from cerebral palsy. International Journal of Innovation and Applied Studies. 2014;9(4):17007.

44.Difazio RL, Vessey JA, Zurakowski D, Snyder BD. Differences in health-related quality of life and caregiver burden after hip and spine surgery in non-ambulatory children with severe cerebral palsy. Dev Med Child Neurol. 2016;58(3):298305.

45. Prudente COM, Barbosa MA, Porto CC. Relation between quality of life of mothers of children with cerebral palsy and the children's motor functioning, After Ten Months of Rehabilitation. Revista Latino-Americana de Enfermagem. 2010;18(2):149-55.

46. Lima MBS, Cardoso V dos S, Silva SS da C. Parental Stress and Social Support of Caregivers of Children With Cerebral Palsy. Paidéia (Ribeirão Preto). 2016;26(64):207-14.

Recibido: 20/01/2019

Aceptado: 01/12/2019 